Understandably, there are considerable differences in methodology and presentation, but all the essential concepts underlying the method are incorporated in Goodwin's tables; the main improvement introduced by Dr. Cunningham (who was, of course, quite unaware of these tables) is the inclusion of tabulations for the azimuth (Journal 17, i 7 ).

Although none of the colleagues to whom I showed my short article called my attention to the existence of Goodwin's tables, I must accept sole responsibility for not having made a more thorough search of the published literature on the subject. This omission does, however, serve to underline the general note in that article on the difficulty of assigning priority for particular methods.

(2) Mr. C. P. Sabelis, Commander of the Nautical College at Den Helder, The Netherlands, has called my attention to an error (due to miscopying) in the equation for the azimuth, which should read

$$
\cos A=\tan \phi \sin \Delta H\left(\mathrm{I}+\frac{1}{2} \tan H_{0} \sin \Delta H+\ldots\right)
$$

The effect is to decrease the stated error in the azimuth.

\title{
Manned Spaced Flight Navigation Techniques
}

\author{
from F. D. P. Wicker
}

I HAVE read with great interest the article entitled 'Manned Space Flight Navigation Techniques' by Major R. C. Henry in the October issue of the Journal.

It would appear that much of the imagined difficulty in interplanetary navigation is due to the fact that until now man has really been concerned with fixing his position and controlling his path on a spherical surface, the third-dimensional problem in air navigation has been one of altitude-i.e. definition of the surface concerned. Now, for the first time, we are involved in what at first sight appears to be a truly three-dimensional problem, and its solution appears difficult, partly because we are to be removed from our familiar graticule of earthly meridians and parallels.

If the problem be divided into two parts, an obvious solution presents itself.

At sea, a ship must be on the surface of the water, and is mainly concerned with its position and course on that surface. An aircraft determines its surface of reference by barometric pressure (which by tradition is termed Pressure Altitude and expressed as a linear measure), and again is then concerned with a twodimensional problem on that surface.

A similar solution presents itself for interplanetary navigation.

The Earth's orbit round the Sun lies in a plane known as the ecliptic, and any space vehicle departing from the Earth must, ipso facto, depart from a point on 
that plane. The Sun also lies on that plane. Each other planet pursues its orbit round the Sun on its own plane (which is in fact its own version of the ecliptic), and of course the Sun lies on the orbital plane of each of the planets. The orbital planes of the planets differ from each other, but since they all pass through the Sun they must intersect, and the angle of intersection is known.

If, for example, we are concerned with a voyage from Earth to Venus, it is obvious that our journey starts from a point on the plane of the ecliptic, and ends at a point on the orbital plane of Venus. By following a trajectory that lies initially in the plane of the ecliptic until that path intersects (as it must) the orbital plane of Venus, and then altering course to our destination on the second plane (see Fig. 1) we can reduce the complexity of our problem. The distance along the trajectory from the Earth at which the orbital plane of Venus will be intersected can be calculated before departure, but it may also be determined gyroscopically. A stable platform, set before departure to lie parallel to Venus's orbital plane, will provide an optical reference for the point of intersection.

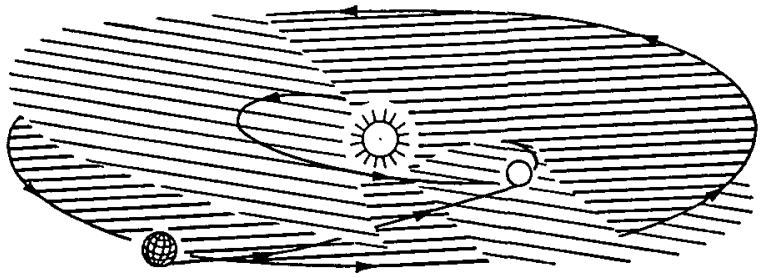

FIG. I.

It will be obvious that this method of flight planning involves two special cases. If departure from Earth is made at one of the nodes, then the trajectory will lie wholly in the orbital plane of Venus; conversely, if the time of arrival is to be when Venus lies on the ecliptic, then the trajectory will lie wholly in that plane. For the first exploratory flights the most opportune time of departure can be arranged. If, in future, support flights are required, a change of course from one orbital plane to the other may be necessary.

This, then, solves the first part of our problem, and provides us with our plane(s) of reference; the second part of the problem is merely the determination of our position on that plane. The obvious (in all senses) point of reference in the solar system is the Sun itself-a point overlooked by Major Henry. The Sun will always be visible on interplanetary flights (unless temporarily eclipsed by another body), and there will be no adverse weather to affect observation. In this case the Sun will be regarded as the nadir, in the same sense as a man standing on the equator regards the centre of the Earth. There is also an old-established reference graticule, namely Sidereal Hour Angle. The position of the space vehicle on the plane of its trajectory may be readily expressed in terms of S.H.A. and distance from the Sun (see Fig. 2).

Sidereal hour angle will be very easy to determine since the stars will all be visible, and their S.H.A.s are already known and tabulated. Distance from the Sun can be determined by measuring the angles subtended by Venus, the Sun and Earth, an exercise in plane trigonometry, since the radii of the planetary orbits are known and also the position of the planets at time of observation. 


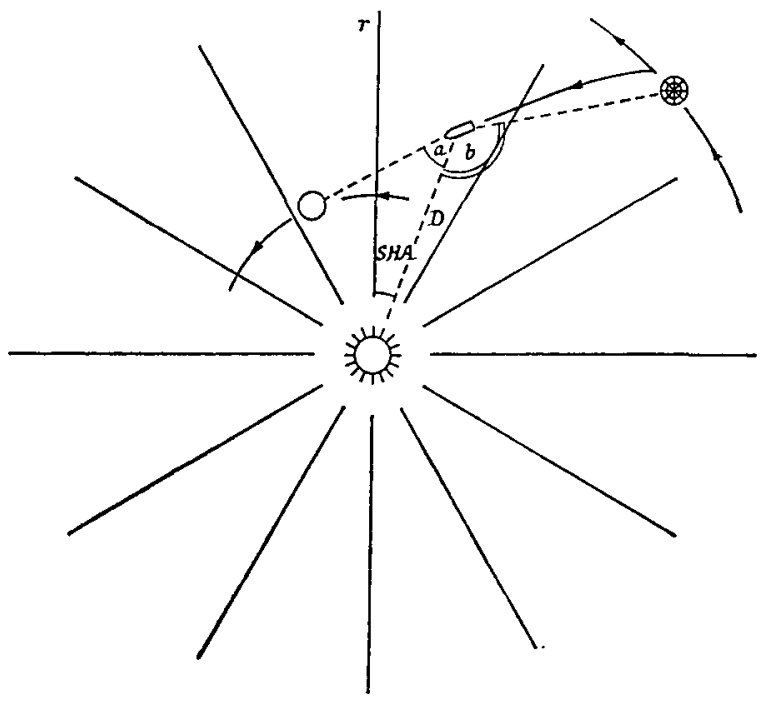

FIG. 2 .

Marine and air navigators are accustomed to use sidereal hour angles whose meridians align with the terrestrial meridians and lie perpendicular to the equinoctial. For the purposes of interplanetary travel it will be necessary to tabulate S.H.A.s with reference to the plane of the trajectory; i.e. in the voyage under example, the meridians of S.H.A. will lie perpendicular to the ecliptic for the first part of the voyage, and to the orbital plane of Venus for the second part. This will involve carrying a table of S.H.A. values and declinations appropriate to each part of the voyage. The transition from one to the other will present no more difficulty than the transition from a conformal conic projection to the Greenwich grid on a polar flight. The change in S.H.A. values for stars near the orbital planes will not be great.

Here it should be observed that a deviation from flight plan along the track will also imply a deviation off the track, since the speed of closing from Earth's orbit to that of Venus will depend on the velocity along the trajectory-which itself determines the centripetal acceleration.

In the special case of a lunar voyage, the trajectory from Earth will lie wholly in the plane of the Moon's orbit round the Earth. The position on that plane can still be established in terms of S.H.A. and Sun distance, but can also be found in terms of angular separation and distance, the latter being determined by stadiametric observations of the diameters of the two bodies.

Finally a word about space rendezvous and docking. A space vehicle orbiting the Earth is in fact following a distorted helix with a curved axis! While a rendezvous on such a path is not impossible, it is somewhat analagous to landing an aircraft cross-wind from an approach at an angle to the centre line of the runway -it can be done but is not to be recommended. When the vehicle orbits the Earth in the plane of the ecliptic the helix has been completely flattened, and all motion is in one plane. Rendezvous and docking with a second vehicle is now much simplified. The passive vehicle should be on the lower orbit, and the approach should be made by the vehicle on the high orbit and docking completed from above. 